\title{
Cooperative binding interaction of ethidium with allosteric DNA
}

\section{Dongchul Suh}

Department of Biochemistry, College of Medicine, Pochon CHA University, Kyungki-Do, 487-800, Korea

Corresponding author: Phone, +82-357-543-2817; Fax, +82+357-543-2818; E-mail, DCSuh@ @anmail.net

Accepted 7 July 1999

Abbreviations: $C D$, circular dichroism; SVD, singular value decomposition

\begin{abstract}
The specific association of drugs with deoxyoligonucleotides, containing a B-Z junction between left-handed ZDNA and right-handed B-DNA, was examined by fluorescence and circular dichroism (CD) technique. Ethidium was chosen for a simple DNA binding compound because it binds to right-handed DNA and hybrid $B-Z$ forms containing a B-Z junction in a highly cooperative manner. The binding isotherms were analyzed by an allosteric model in order to describe the cooperativity of association. Binding of ethidium to the DNA that are initially in the hybrid B-Z forms showed over an order of magnitude higher affinity than other DNA which were entirely in the B-form. The conformational transitions of deoxyoligonucleotides containing a B-Z junction as a result of ethidium binding were monitored by $C D$ and the influence of $\mathrm{NaCl}$ on the complex formation was also determined by the CD spectra. The singular value decomposition (SVD) analysis was used to characterize a family of CD spectra of the species in binding equilibria. The results of SVD analysis showed a strikingly complex thermodynamic equilibria of cooperative binding of drugs to the allosterically converted DNA forms. The results also showed that these DNA forms in low- and high-salt were different in the absence or presence of drug. These results demonstrate that DNA- binding-drugs can preferentially interact with specific DNA structures and that these interactions are accompanied by allosteric changes of DNA conformations.
\end{abstract}

Keywords: allosteric DNA, cooperativity, CD spectra, singular value decomposition, binding interactions

\section{Introduction}

DNA has been shown to adopt different conformations depending on its sequence and environment. This unique structural feature of drug binding specificity with unique DNA structures provide the potential targets for antibiotics or antitumor drugs Walker et al., 1985; (Suh et al., 1991; Suh, 1996). DNA is known to induce conformational changes in localized sites as well as in the entire secondary structures. In the localized conformational transitions, unusual DNA structures (bends, bulges, junction) may appear (Wells, 1988). Such structures may be recognized by proteins or antibiotics by unwinding or unstacking DNA double strand helix. These disruptions of stacked base pairs lower the energy barrier at the binding sites in the event of binding interaction. A detailed understanding of the DNA binding properties of DNA binding compounds is essential for the rational design of new DNA binding agents (Haq et al., 1995; Suh \& Chaires, 1995; Chaires et al., 1996; Suh et al., 1997). In order to describe thermodynamic effects in unusual DNA structures, several models requiring the presence of junction formation between two different secondary structures in contiguous DNA segments have been applied (Burd et al., 1975; Crothers \& Fried, 1983). Junctions can comprise only small regions of long DNA sequences because they are thermodynamically unstable. Since Z-DNA can exist in vivo (Wittig, 1989) and may be related to gene regulation (Jimenez-Ruiz et al., 1991), the characteristics of the B$Z$ junction have been of interest to investigators.

The nucleotide sequence of the first of these synthesized, designated BZ-1, has been characterized to focus on the unusual localized conformational transitions which may occur infrequently in natural genomes. Recently, the structure, energetics, thermodynamics, and reactivity of B-Z junctions have been reported (Sheardy, 1988; Sheardy et al., 1993; Sheardy et al., 1994; Suh, 1996). From these studies, it was found that the formation of a junction requires more than $1 \mathrm{Kcal} /$ mol and is dependent on sequence. The B-Z junction region spans $3 \mathrm{bp}$ long and perturbs the structure of flanking right-handed helix regions. Ethidium was chosen as a simple DNA binding compound because it binds tight to the allosterically converted DNA form in high salt conditions which initially favors the hybrid B-Z form than any other forms (Suh et al., 1991). In order to reveal the nature of highly reactive DNA forms, $C D$ spectra of ethidium bound BZ-1 were collected for the conformational transition, and analyzed by singular 
value decomposition. These results demonstrate that DNA binding drugs can preferentially interact with specific DNA structures and that these interactions are accompanied by allosteric changes of DNA conformations.

\section{Materials and Methods}

\section{Materials}

A deoxyoligonucleotide, which is designated $B Z-1$, is synthesized on an Applied Biosystems 380B DNA Synthesizer using the phosphoramidite method (Caruthers, 1991) and purified by trityl-selective reverse-phase HPLC, as previously described (Sheardy, 1988). The sequence of $\mathrm{BZ}-1$ is as follows:

\section{5'-CGCGCGCGACTGACTG 3'-GCGCGCGCTGACTGAC}

where $C$ in italic indicates 5-methyl-cytosine. Each strand of complementary deoxyoligonucleotide is mixed at equal concentration, heated up to $90^{\circ} \mathrm{C}$ and then slowly cooled down to $20^{\circ} \mathrm{C}$, in order to anneal the non, selfcomplementary sequences. A complete annealing has been determined by the continuous variation method (Huang, 1982; Haq et al., 1995), so called, Job plot (data not shown). The extinction coefficient has been obtained by calculating that of each deoxymononucleoside monophosphate (Fasman, 1975), and by measuring the percent of hyperchromicity of oligonucleotides. The measured extinction coefficient was used for concentration determinations. Ethidium was purchased from Sigma (St. Louis, MO). Ethidium concentrations were determined by absorbance, at $480 \mathrm{~nm}$ assuming $\varepsilon=5,600 \mathrm{M}^{-1} \mathrm{~cm}^{-1}$. Experiments were performed in BPE buffer (low-salt) containing $6 \mathrm{mM} \mathrm{Na} 2 \mathrm{HPO}_{4}, 2 \mathrm{mM}$ $\mathrm{NaH}_{2} \mathrm{PO}_{4}$, and $1 \mathrm{mM}$ EDTA, pH 7.0. The BPE buffer plus $4.5 \mathrm{M} \mathrm{NaCl}$ (high-salt) was prepared by adding stock $\mathrm{NaCl}$ solution to the BPE buffer at $4.5 \mathrm{M} \mathrm{NaCl}$.

\section{Spectral Measurement}

Absorbance was measured at $20^{\circ} \mathrm{C}$, with a Cary- 219 spectrophotometer. The cell path length of spectrophotometer was $1 \mathrm{~cm}$. Circular dichroism was measured at $20^{\circ} \mathrm{C}$ with a JASCO J-500A spectropolarimeter, interfaced to and controlled by an PC. The cell path length of $C D$ measurements was $1 \mathrm{~cm}$.

\section{Fluorescence Titrations}

Ethidium binding was monitored by a steady-state fluorescence measurements on a Perkin-Elmer 650 spectroflorometer, which was not equipped with a polarizer. The wavelength of excitation was $525 \mathrm{~nm}$, and that of emission was $605 \mathrm{~nm}$. Fluorescence titrations were described in detail before (Suh et al., 1991; Suh, 1996). The bound ethidium concentration $\left(C_{b}\right)$ was calculated from the relation,

$C_{b}=C_{t}\left(I-I_{0}\right) /(V-1) I_{0}$.

where $C_{t}$ is the total ethidium concentration, $I$ is the observed fluorescence intensity, $I_{0}$ is the fluorescence intensity of the same concentration of ethidium in the absence of DNA, and $V$ is the experimental coefficient determined in BPE buffer and in BPE at $4.5 \mathrm{M} \mathrm{NaCl}$. Free ethidium concentration $\left(\mathrm{C}_{\mathrm{f}}\right)$ were obtained from the conservation relationship, $C_{f}=C_{t}-C_{b}$. The binding ratio, $r$ is defined as $r=C_{b} /[D N A]$. The fluorescence intensity of ethidium was calibrated for the inner-filter effect, particularly when the fluorescence titrations were performed at high concentration of total added ethidium (Lakowicz, 1983).

\section{Monitoring the conformational changes of DNA}

The conformational transition of BZ-1 by the effect of ethidium binding was monitored by circular dichroism measurements. Each $1 \mathrm{ml}$ solution of BZ-1 in low- and high-salt, respectively, was prepared and was equilibrated for overnight in the dark place. Ethidium was added, ranging from $1.3 \mu \mathrm{M}$ to $20.8 \mu \mathrm{M}$, to 9 separate solutions at the final concentration of $38 \mu \mathrm{M}$ (bps) in low and high salt, respectively. Independently, CD spectra of $\mathrm{NaCl}$ induced conformational transition of ethidium bound BZ-1 were measured. Each $1 \mathrm{ml}$ solution of 18 $\mu \mathrm{M}$ ethidium ranging from 0 to $4.5 \mathrm{M} \mathrm{NaCl}$ was prepared, separately. DNA was added to each solution at a concentration of $38 \mu \mathrm{M}$ (bps) and equilibrated for overnight in the dark place. CD spectra of a family of samples were recorded, while some aliquots were taken out to measure the fluorescence intensity, independently, in order to estimate the bound ethidium concentration. Simultaneously, the fluorescence intensity of same ethidium concentration solution was measured in the absence of DNA.

A family of $C D$ spectra was analyzed by singular value decomposition (SVD), which was available in PCMATLAB (The Mathworks, Inc., Natick, MA). The wavelengths used for SVD analysis were spanned in the range of 220-360 nm at $2 \mathrm{~nm}$ intervals shown in Figure 3 and Figure 5.

\section{Results and Discussions}

\section{Salt-dependent CD spectra of BZ-1}

Representative CD spectra of BZ-1 collected as a function of $\mathrm{NaCl}$ concentration are shown in Figure 1. CD spectra of BZ-1 shows development of a hybrid structure at high salt and the magnitude of the molar ellipticity decreases with increasing $\mathrm{NaCl}$. The $\mathrm{G}-\mathrm{C}$ end of the molecule adopts the left-handed Z-DNA and the central $3 \mathrm{bp}$ region forms a stable junction region as demonstrated by NMR. The rest of the molecule adopts 


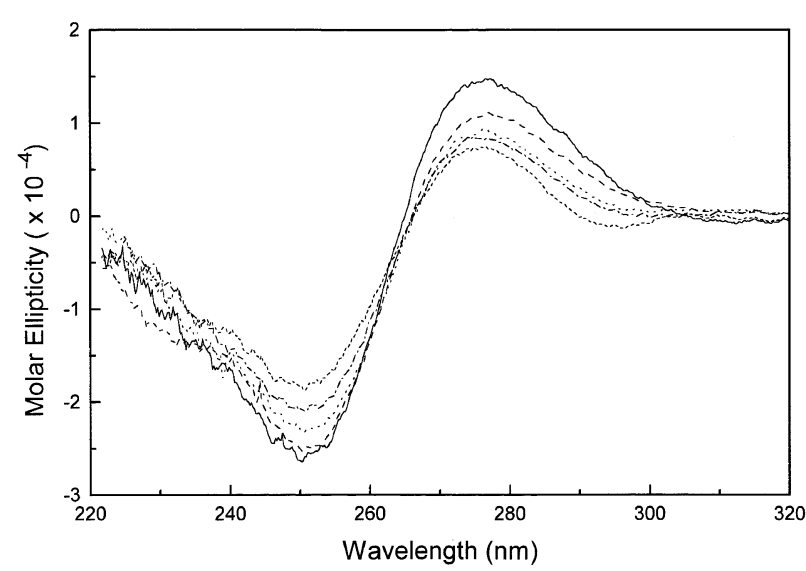

Figure 1. Circular dichroism spectra of $\mathrm{BZ}-1$ as a function of $\mathrm{NaCl}$ concentration: solid line (_) represents a spectrum at low salt; dotted line (....) represents a $C D$ spectrum at high salt.

the right-handed $B$ conformation. Changes in the $C D$ spectrum of BZ-1 are followed by the conformational transition of the duplex form, a right-handed B-form to a hybrid form containing a B-Z junction. The isoelliptic point can be observed near $260 \mathrm{~nm}$. Although the isoelliptic point seems to be well defined the transition may not be a simple two-state process (Sheardy et al., 1993)

\section{Ethidium binding to $\mathrm{BZ}-1$}

We have earlier reported that fluorescence was strongly enhanced by binding of ethidium to DNA (Suh et al., 1991). In this study, the binding of ethidium to BZ-1 examined by a steady state fluorescence titration was carried out at fixed DNA concentration with increasing amounts of ethidium concentrations. Scatchard plot of the binding data plotted by calculating the free and the bound ethidium concentrations is shown in Figure 2. The neighbor-exclusion model (McGhee \& von Hippel, 1974) designed for the cooperative binding analysis was first used for estimation of the cooperative binding isotherms. However, this neighbor-exclusion model, showed poor fitting of the binding data of ethidium with BZ-1. Binding isotherms are dramatically deviated from the calculated lines by the neighbor-exclusion model shown in Figure 2. Thus, the allosteric binding model that have also been used for the quantitative analysis of binding data (Dattagupta et al., 1980; Chaires, 1986), was tried. This model can be used to describe the association of ligand at the equilibrium state of the conformational transition between two different forms of DNA. This equilibrium requires two equilibrium constants, $\sigma$ for a nucleation step and s for a propagation step. The ligand may bind to each DNA form. Neighbor exclusion parameters $\mathrm{K}_{\mathrm{i}}, \mathrm{n}_{\mathrm{i}}$, and $\omega_{\mathrm{i}}$, where $\mathrm{i}=1$ or 2 , represent each DNA form, respectively. The solid lines are the curves derived by an allosteric model (Figure 2). The parameter estimates of the allosteric interactions of

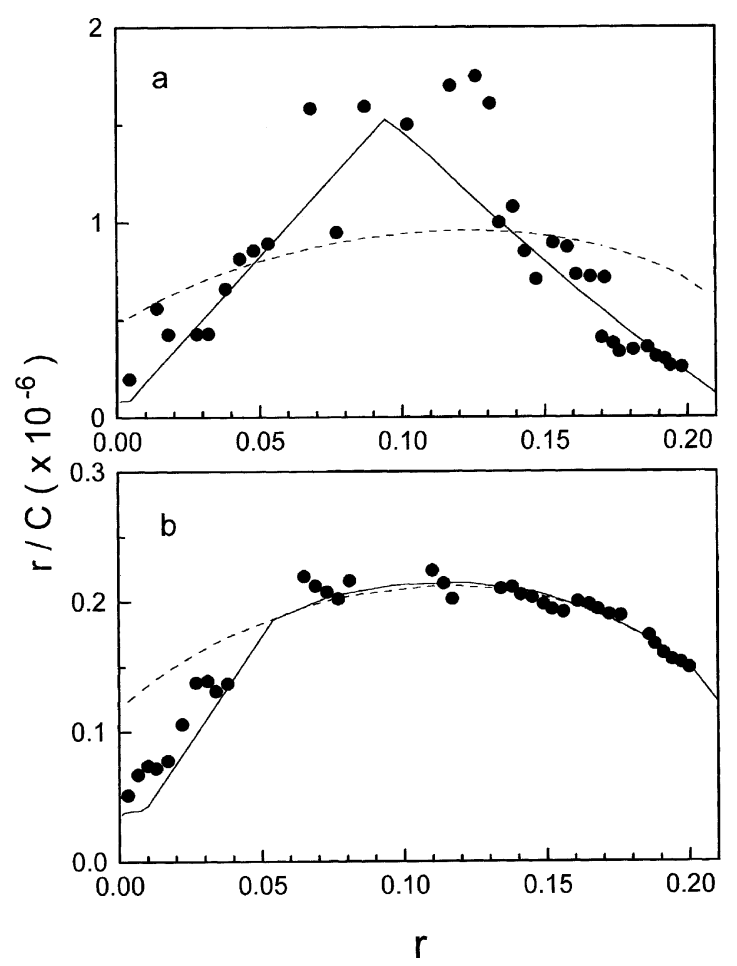

Figure 2. Binding isotherms of ethidium with $B Z-1$ in the form of Scatchard plot. (a) In BPE at 4.5 M NaCl. (b) In BPE buffer. Solid lines represent the calculated curves of an allosteric model. Dotted lines are the least-squares fits of data to the neighbor exclusion model. $C$ is the free ethidium concentration and $r$ is the binding ratio. Summary of the parameter estimates for an allosteric model was reported before (Suh, 1996).

ethidium with BZ-DNA were described previously (Suh, 1996). Ethidium was shown to bind 70 times tighter to the form-2 DNA in high salt, which initially favors formation of the hybrid $B-Z$ form, than to the $B$ form in low salt.

\section{Conformational transition of BZ-1 by the effect of ethidium}

CD spectra of BZ-1 were measured at two $\mathrm{NaCl}$ concentrations by varying the amount of ethidium shown in Figure 3 . These spectra represent the conformational transition of BZ-1 by the effect of ethidium binding. Ethidium was added in increments of $0.06 \mathrm{~mol}$ per bp over the range of 0 to 0.24 . In low salt, we observed the induced CD bands (see Figure $3 a$ ) in the 310-360 nm region. Similar results were observed with natural DNA (Chirico et al., 1990) and with synthetic DNA polymers (Walker et al., 1985). These induced CD bands were suggested as the result of direct interactions between ethidium molecules bound in neighboring binding sites (Aktipis \& Kindelis, 1973). However, there were no significant induced $C D$ bands of spectra in high salt (Figure $3 b$ ). These spectra were thoroughly examined by singular value decomposition (SVD) analysis that have been applied for obtaining the number of 

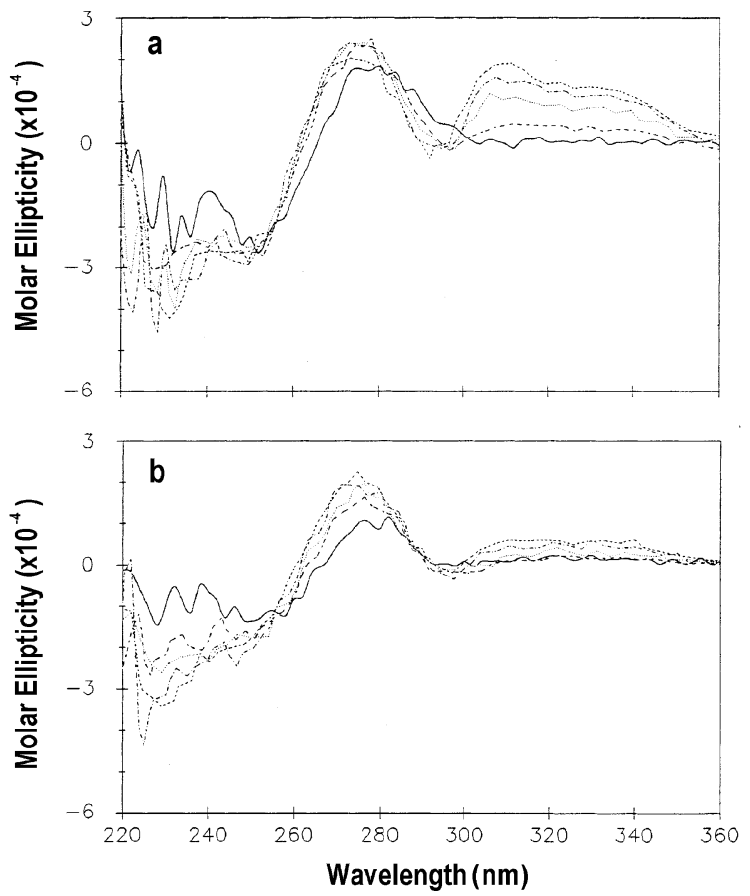

Figure 3. Some representative $C D$ spectra for the interaction of ethidium with BZ-1 at two $\mathrm{NaCl}$ concentrations. (a) In BPE buffer. (b) In BPE at 4.5 $\mathrm{M} \mathrm{NaCl}$. The spectra representing as solid lines were at low binding ratio, while those as dotted lines were at high binding ratio. Ethidium titration were obtained in increments of about $2.5 \mathrm{mM}$ ethidium at each time.

significantly independent components in a set of spectra (Henry \& Hofrichter, 1992; Johnson, 1992). A family of $\mathrm{CD}$ spectrum for $\mathrm{NaCl}$-induced transition from the righthanded $\mathrm{B}$ form to a hybrid form containing a B-Z junction was analyzed by SVD (Sheardy et al., 1993). The transition was noted: it is at least three-state process including the intermediate process (I), shown in the central region of Figure 7.

In brief, the data matrix $A$ can be decomposed into the product of three matrices, $A=U S V^{\top}$, where the $S$ matrix represents the singular value, the $U$ and $V$ matrices are called a matrix of basis vectors $(U)$ and a matrix of amplitude coefficients $(\mathrm{V})$, respectively. The $V$ matrix contains coefficients for the least-squares fit the basis spectra which is the product, US, to the original CD spectra. The original CD spectra are in the range of 220-360 nm at $2 \mathrm{~nm}$ intervals. The CD spectra in Figure 3 and Figure 5 are composed to produce the data matrix A. Among the matrices, the number and the magnitude of significant singular values in the $S$ matrix, can be used for selecting the most significant components. The two most significant basis CD spectra of BZ1 by the effect of ethidium in low and high salt are shown in Figure $4 a$ and $b$, respectively. The only two largest singular values represent these sets of spectra analyzed by SVD. Other basis spectra seem to be randomly distributed with noise of signals. The solid lines represent the most significant basis CD spectra in low and high salt, which are different in their magnitudes of spectra particularly at $280 \mathrm{~nm}$ and in the range of 300-360 $\mathrm{nm}$. The dashed lines represent the second most significant basis spectra in low and high salt which are very similar in their magnitudes and shapes as a function of wavelength. It has been discussed that the most significant basis spectrum shows the common features in a series of spectra under the same condition (Johnson, 1992). Thus, these most significant basis spectra can best describe for each spectrum in a series of $C D$ spectra as the single shape under the same condition. The amplitude coefficients for the most significant components in low and high salts are shown in Figure $4 c$ and $d$, respectively. The filled circles represent the coefficients for the most significant spectra and the empty circles represent those for the second most significant spectra. The amplitude coefficients for the most significant basis spectra changes little at the beginning, then increases slowly by adding ethidium and finally stays constant. Comparing these behaviors, the coefficients for the second most significant components change little at the beginning, but increases dramatically during the titration of ethidium and then finally stays constant. The amplitude coefficients of the rest corresponding basis spectra are randomly distributed like noise.

Another criterion for the selection of the significant components is to assess the signal to noise ratio of the columns of $U$ and $V$. The autocorrelation function, $C(R i)$ can be used to assess quantitatively the randomness of corresponding columns of the $U$ and $V$ matrix to columns of each singular value; $C\left(R_{i, j}\right)=\Sigma\left(C\left(R_{i}\right) C\left(R_{j}\right)\right)$, where $\mathrm{R}_{\mathrm{j}, \mathrm{i}}$ represent the $\mathrm{j}$-th elements of the $\mathrm{i}$-th columns of $U$ or $V$, respectively. Those column vectors are normalized to unity. If those vectors show slow variations from row to row, "signal", the autocorrelation values are close to 1 and if those show rapid variations, "noise", they are close to -1 . The autocorrelation values of the first and second element of basic vectors stay above 0.8 , but those values below third elements falls below 0.3. Similarly, the values of the first and second elements of amplitude vectors are above 0.5 and then values of other elements falls down to be negative as the values of 'noise'. Johnson suggested that if the autocorrelation values were greater than 0.3 , those elements were significant, but if these were smaller than 0.1 or negative, those were not significant (Johnson, 1992). Thus, SVD analysis shows that the B and the hybrid B-Z form to ethidium bound forms transition in low and high salts, respectively is a simple two-state process. The similarity of induced CD bands also represents a close similarity of geometry of DNAethidium complexes (Aktipis \& Martz, 1974). There is no such similarity between the induced CD bands in low and high salts. 

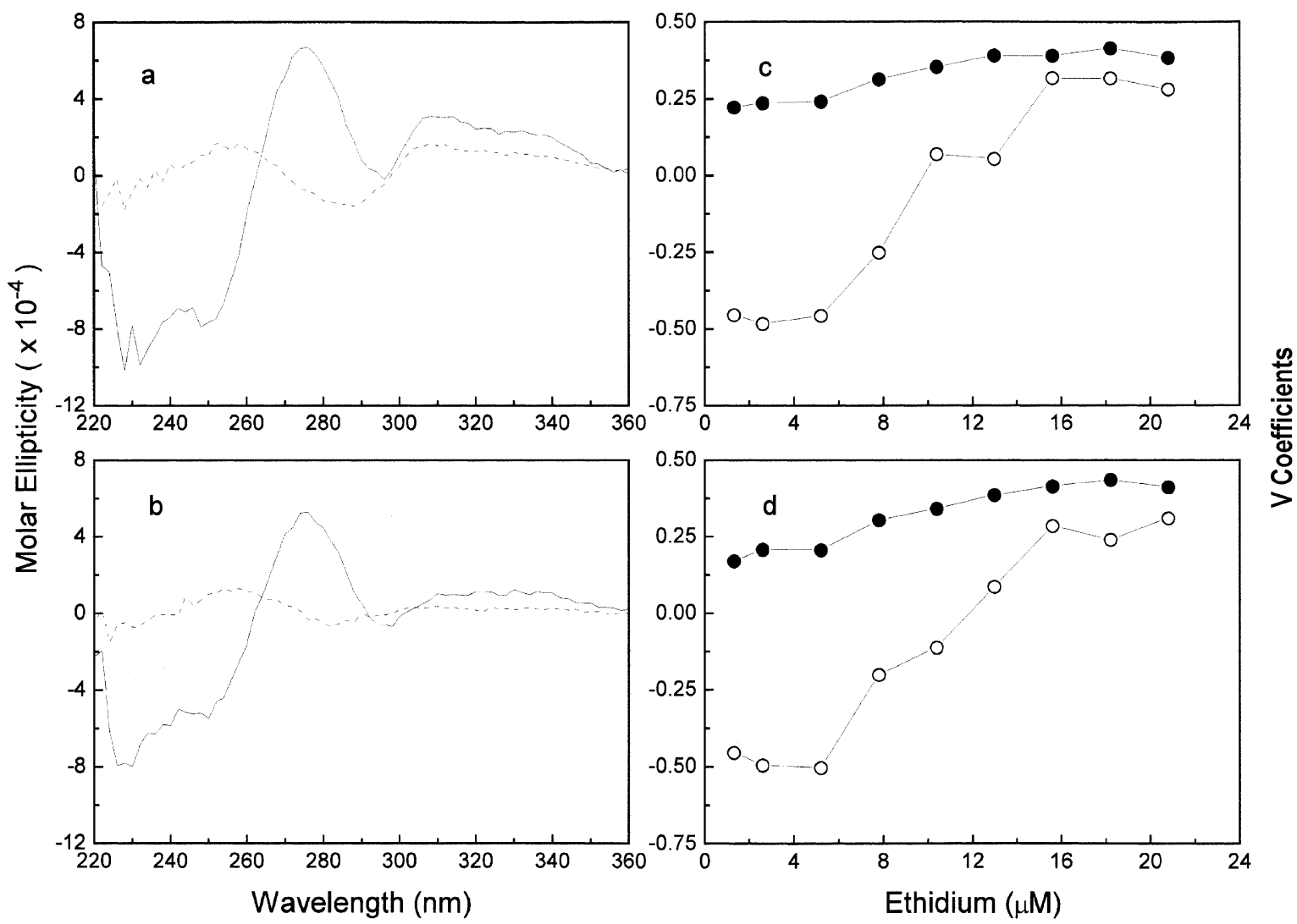

Figure 4. The two most significant basis spectra and amplitude vectors for the interaction of ethidium with $\mathrm{BZ}-1$ at two $\mathrm{NaCl}$ concentrations analyzed by singular value decomposition. Basis spectra (a) in BPE buffer, (b) in BPE buffer at $4.5 \mathrm{M} \mathrm{NaCl}$, and amplituide vectors (c) in BPE buffer, and (d) in BPE buffer at $4.5 \mathrm{M} \mathrm{NaCl}$. In both panels, the solid lines show the most significant basis spectra and the dashed lines show the second most significant basis spectra. Filled circles are amplitude vector coefficients of components- 1 and open circles are those of components- 2 .

\section{Monitoring the conformations of ethidium bound unusual DNA by $\mathrm{NaCl}$}

In an effort to understand these characteristics clearly, the saturating amount of ethidium was added to BZ-1 by maintaining the binding ratio of 0.25 . Then, $\mathrm{NaCl}$ was added up to $4.5 \mathrm{M}$ and compared changes in $\mathrm{CD}$ spectra (Figure 5). The magnitude of $C D$ spectra decreases by increasing $\mathrm{NaCl}$ concentration particularly in the range of $300-360 \mathrm{~nm}$. The $\mathrm{CD}$ band in a range of $290-360 \mathrm{~nm}$ is the representative characteristic of direct interaction between ethidium molecules bound in neighboring bind-ing sites (Aktipis \& Kindelis, 1973). The characteristics in this region of CD spectra clearly indicate the clustering of bound drugs. The binding of ethidium to the hybrid $B-Z$ form in high salt shows difference where the bound molar ellipticity increases up to 8 at the saturated level of ethidium (Suh, 1996). Earlier report of Aktipis and Kindelis (1973) showed that salt had no effect on the dependency of bound ellipticity for ethidium with calf thymus DNA. The CD spectra analyzed by SVD indicated that first, three significant spectral species may exist as determined by the singular values. These three basis $C D$ spectra are shown in

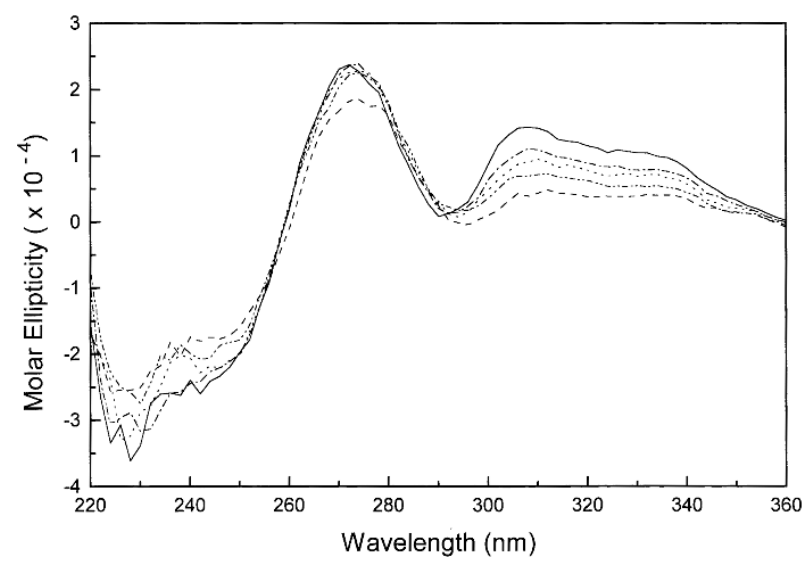

Figure 5. CD spectra represented for the interaction of saturating amount of ethidium with $\mathrm{BZ}-1$ by varying $\mathrm{NaCl}$ concentration. The magnitude of $\mathrm{CD}$ spectra decreases with increasing $\mathrm{NaCl}$ concentration, especially in the range of 290-350 nm. The spectrum as a solid line is in BPE buffer, while the spectrum as a dotted (----) line were obtained in BPE buffer at $4.5 \mathrm{M}$ $\mathrm{NaCl}$.

Figure $6 a$ and $b$. The solid line (see Figure 6a) represents the second most significant basis spectrum 

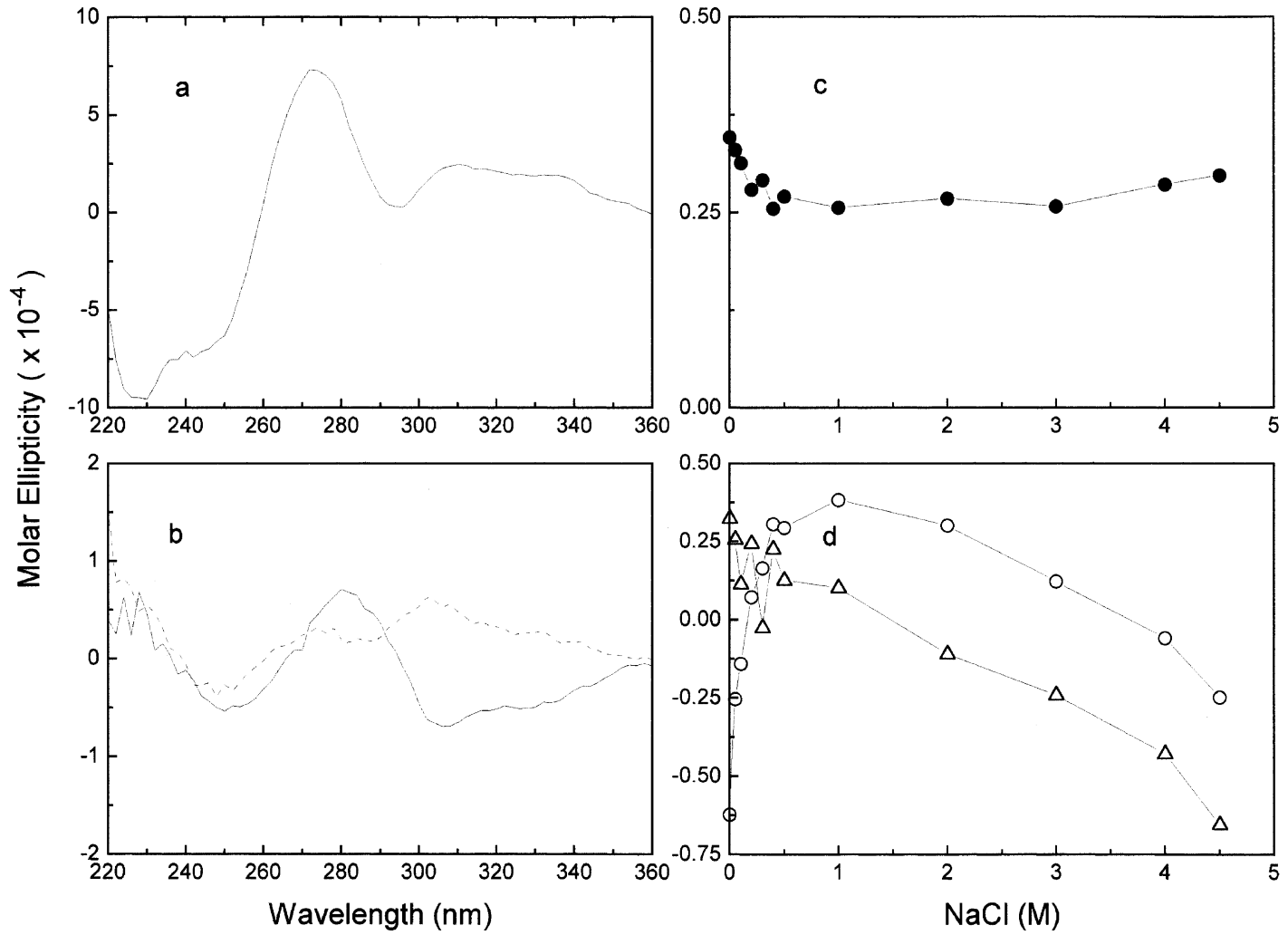

Figure 6. The three most significant basis spectra and amplitude vectors for the interaction of saturating amount of ethidium with $\mathrm{BZ}-1$ as a function of $\mathrm{NaCl}$ concentration analyzed by singular value decomposition. (a) The most significant basis spectrum. (b) The solid line shows the second and the dashed line shows the third most significant spectrum. (c) The amplitude vector of the most significant component. (d) The empty circles represent the amplitude vector of the second most significant component and empty triangle represent that of the third most significant component.

which is, however, very much different from that in Figure 6a. The dashed line shows the third basis spectrum in Figure $6 \mathrm{~b}$. In conjunction with these basis spectra, there are three significant amplitude vectors shown in Figure $6 \mathrm{c}$ and $\mathrm{d}$. The first most important amplitude vector is decreased slightly initially but did not change much as a function of $\mathrm{NaCl}$ concentration in Figure 6c. The second, repre-sented as empty-circles, increases sharply up to $1 \mathrm{M}$ and then slowly decreases while the third, shown as empty-triangles decreases continually as a function of $\mathrm{NaCl}$ concentration. Other amplitude vectors are randomly distributed (data not shown). The autocorre-lation values of both vector decreases sharply below 0.1 . Therefore, SVD analysis data show that the transition of the ethidium-saturated DNA complex by salt seems to be a complex process involving sequential three-state process instead of a simple two-state process. The structure of ethidium bound BZ-1 in low and high salt, respectively, seems clearly not to be the same because of the dramatically different $C D$ spectrum.

Thermodynamic pathways of binding equilibria exist A minimal set of equilibria, which can describe binding data and CD results, is shown in Figure 7 . There are two major species; one in low salt (B-DNA \& B'-DNA) and the other in high salt (BZ-DNA \& B"-DNA) in the presence of ethidium as described earlier by the use of an allosteric model for fitting the binding data of ethidium with BZ-1 (Figure 2). The existence of two major species in the presence of a drug is consistent with two minimal species of equilibria by an allosteric model of binding data. Ethidium binds to both conformational forms. The nature of most of DNA forms is unknown, except that of form-1 DNA (B-DNA) in low salt and form2 DNA (BZ-DNA) in high salt, respectively. Secondly, there are at least three major species of BZ-1 in the absence [results of SVD analysis were reported before (Sheardy et al., 1993)] and the presence of drug (shown in Figure $5 \& 6$ ) and by the effect of $\mathrm{NaCl}$. The nature of intermediate (I or l') may be an unfavored nucleated species for the $B$ to $B-Z$ transition. Similar behaviors were reported for the $\mathrm{B}$ to $\mathrm{Z}$ transition before (Jovin et al., 1987). It is probably formed because the WatsonCrick hydrogen bonds are broken by an unwinding of the double helix, likely to happen during the $B$ to $Z$ transition (Manzini et al., 1987). Finally, a minimal equilibria in Figure 7 can be explained for $B$ to $B Z$ transition and for the cooperative conversion of DNA with drugs as described above. 
Low-salt

High-salt

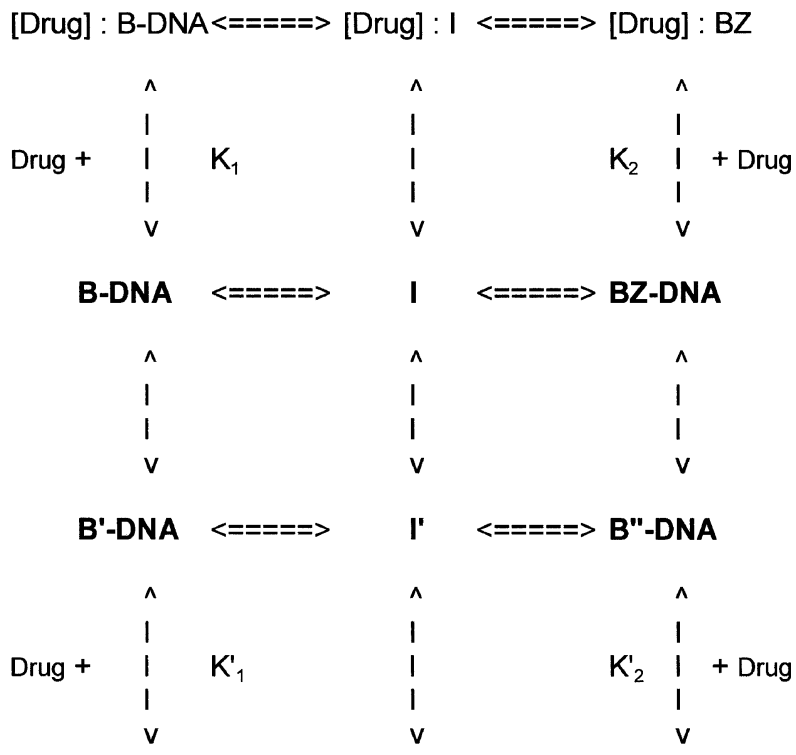

[Drug] : B'-DNA<=====> [Drug] : l' <=====> [Drug] :B"

Low-salt

High-salt

Figure 7. Minimal equilibria for describing the cooperative binding of drug to allosterically converted DNA forms. Details are described in the text.

\section{Acknowledgements}

This work was supported by the research grant (Genetic Engineering Project \#: 1998-019-D00083) from Korean Ministry of Education. BZ-1 was kindly given by Dr. Sheardy (Seton Hall University, USA). I would like to thank Dr. Chaires (The University of Miss. Medical Center, USA) for helpful discussions.

\section{References}

Aktipis, S. and Kindelis, A. (1973) Optical Properties of the Deoxyribonucleic acid - ethidium bromide complex. Biochemistry 12: 1213-1221.

Aktipis, S. and Martz, W. W. (1974) Circular Dichroism and Temperature-optical Density Studies on the Conformation of Polynucleotide-ethidium bromide Complex. Biochemistry 13: 112-118.

Burd, J. F., Wartell, R. M., Dodgson, J. B. and Wells, R. D. (1975) Transmission of Stability in Deoxynucleotides. J. Biol. Chem. 250: 5109-5113.

Caruthers, M. H. (1991) Chemical Synthesis of DNA and DNA Analogues. Acc. Chem. Res. 24: 278-284.

Chaires, J. B. (1986) Allosteric Conversion of Z-DNA to an Right-handed Conformation by Daunomycin. J. Biol. Chem.
261: 8899-8907.

Chaires, J. B., Saty, S., Suh, D., Fokt, I., Przewloka, T. and Priebe, W. (1996) Parsing the Free Energy of Anthracycline Antibiotic Binding to DNA. Biochemistry 35: 2047-2053.

Chirico, G., Lunelli, L. and Baldini, G. (1990) Conformation of Intercalated DNA Plasmids Investigated by Circular Dichroism and Dynamic Light Scattering. Biophys. Chem. 38: 201-211.

Crothers, D. M. and Fried, M. G. (1983) Transmission of Longrange Effects in DNA. Cold Spring Harbor Symp. Quant. Biol. 47: 263-269.

Dattagupta, N., Hogan, M. and Crothers, D. M. (1980) Interaction of Netropsin and Distamycin with DNA. Biochemistry 19: 5998-6005.

Fasman, G. (1975) Measured and Calculated Extinction Coefficient at 260nm, 25C, and Neutral pH for Single-Strand RNA and DNA, in: CRC Handbook of Biochemistry and Molecular Biology, $3^{\text {rd }}$ Ed., CRC Press, Cleveland.

Haq, I., Lincoln, P., Suh, D., Norden, B., Chowdhry, B. Z. and Chaires, J. B. (1995) Interaction of D- and $L-\mathrm{Ru}^{2+}$ with DNA: A Calorimetric and Equilibrium Binding Study. J. Am. Chem. Soc. 117: 4788-4796.

Henry, E. R. and Hofrichter, J. (1992) SVD Application to Analysis of Experimental Data in: Methods in Enzymology, vol. 210, (Brand, L. and Johnson, M.L., eds.), pp.129-192, Academic Press, Inc., San Diego.

Huang, C .Y. (1982) Determination of Binding Stoichiometry by the Continuous Variation Method: The Job Plot in: Methods in Enzymology, vol. 87, pp.509-525, Academic Press, Inc., San Diego.

Jimenez-Ruiz, A., Requena, J. M., Lopez, M. C. and Alonso, C. (1991) A Potential Z-forming DNA is Located Between Two Transcription Units Alternatively Expressed During Development of Drosophila hydei. Proc. Natl. Acad. Sci. USA, 88: 3135.

Johnson, W. C., Jr. (1992) Analysis of CD Spectra in: Methods in Enzymology, vol 210, (Brad, L. \& Johnson, M.L. eds.) pp.426-447, Academic Press, Inc., San Diego.

Jovin, T. M., Soumpasis, D. M. and McIntosh, L. P. (1987) The Transition Between B-DNA and Z-DNA. Ann. Rev. Phys. Chem. 38: 521-560.

Lakowicz, J. R. (1983) Chapt.2: Instrumentation for Fluorescence Spectroscopy, In: Principles of Fluorescence Spectroscopy, Plenum Press, New York.

Manzini, G., Xodo, L. E., Quadrifoglio, F., van Boom, J. H. and van der Marel, G. A. (1987) dG-dC Alternating Oligonucleotides: Thermodynamics and Kinetic Aspects of the B-Z Transformation. J. Biomol. Struct. Dyn. 4: 651-662.

McGhee, J. D. and von Hippel, P. H. (1974) Theoretical Aspects of DNA-Protein Interaction. J. Mol. Biol. 86: 469-489.

Sheardy, R. D. (1988). Preliminary Spectroscopic Characterizations of a Synthetic DNA Oligomer Containing a B-Z Junction at High Salt. Nucleic Acids Res. 16: 1153-1167.

Sheardy, R. D., Suh, D., Kurzinsky R., Doktycz, M. J., Benight, 
A. S. and Chaires, J. B. (1993) Sequence Dependence of the Free Energy of B-Z Junction Formation in Deoxyoligonucleotides. J. Mol. Biol. 231: 475-488.

Sheardy, R. D., Levine, N., Marotta, S., Suh, D. and Chaires, J. B. (1994) A Thermodynamic Investigation of the Melting of B-Z Junction Forming DNA Oligomers. Biochemistry 33: 1385-1391.

Suh, D., Sheardy, R. D. and Chaires, J. B. (1991) Unusual Binding of Ethidium to a DNA Containing a B-Z Junction. Biochemistry 30: 8722-8726.

Suh, D. and Chaires, J. B. (1995) Criteria for the Mode of Binding of DNA Binding Agents. Bioorg. Med. Chem. 3: 723728.

Suh, D. (1996) Specific Recognition of Unusual DNA Structures by Small Molecules: An Equilibrium Binding Study.
J. Biochem. \& Mol. Biol. 29: 1-10.

Suh, D. and Povirk L. F. (1997) Mapping of the CleavageAssociated Bleomycin Binding Site on DNA with a New Method Based on Site-Specific Blockage of the Minor Groove with $\mathrm{N}^{2}$-Isobutyrylguanine. Biochemistry 36: 4248-4257.

Walker, G. T., Stone, M. P. and Krugh, T. R. (1985) Ethidium Binding to Left-handed DNA Results in Regions of Righthanded DNA at the Intercalation Site. Biochemistry 24: 74627471.

Wells, R. D. (1988) Unusual DNA Structures. J. Biol. Chem. 263: $1095-1098$.

Wittig, B., Dorbic, T. and Rich, A. (1989) The Level of Z DNA in Metabolically Active, Permeablized Mammalian Cell Nuclei is Regulated by Torsional Strain. J. Cell Biol. 108: 755-764. 